\title{
ON GROUPS OF DIFFEOMORPHISMS
}

\author{
T. E. STEWART
}

I. We consider here the groups of homeomorphisms on Euclidean $n$-space and the $n$-sphere $S^{n}$. Chiefly we will be concerned with the question of whether or not these groups reduce in an homotopy sense to the ordinary orthogonal group acting on these spaces. Such questions are intimately connected with the theory of fibre bundles in which these spaces occur as fibres. We will restrict ourselves to the case where the homeomorphisms are of class $C^{1}$ and will topologize the various groups taking account of the differentiability.

We first consider Euclidean $n$-space $E^{n}$. We denote by $K$ the group of all homeomorphisms $f$ of $E^{n}$ such that $f$ and $f^{-1}$ are of class $C^{\prime}$. $K$ becomes a topological group by demanding uniform convergence of $f$ and its derivatives on compact sets, ${ }^{1}$ i.e. a typical neighborhood of the identity function is given by

$$
\begin{array}{r}
V_{r, \mathrm{e}}=\left\{g \in K|\|g(x)-x\|<\epsilon,| \frac{\partial g_{i}}{\partial x_{k}}(x)-\delta_{k}^{i} \mid<\epsilon,\|x\|<r\right. \\
i, k=1, \cdots, n\} .
\end{array}
$$

Clearly the orthogonal group, $O_{n}$, of $E^{n}$ is imbedded in $K$ and the topology induced on $O_{n}$ is the usual topology. In fact any locally compact or complete metric group which acts as a transformation group of $E^{n}$ so that each motion is of class $C^{1}$ is imbedded in $K$ (see $[1$, p. 197]). With this topology on $K$ we now show

Theorem I. $O_{n}$ is a deformation retract of $K$.

First we will demonstrate a number of lemmas which lead to the proof of Theorem I.

Lemma 1. Let $K_{0}=\{g \in K \mid g(0)=0\}$. Then $K_{0}$ is a closed subgroup of $K$ and $K$ decomposes topologically into $E^{n} \times K_{0}$. Hence if $O_{n}$ is a deformation retract of $K_{0}$, then $O_{n}$ is a deformation retract of $K$.

Proof. With each $x \in E^{n}$ associate $\sigma_{x} \in K, \sigma_{x}(y)=y+x$. Clearly $x \rightarrow \sigma_{x}$ is topological. If $f \in K$, consider $f(0)=x_{0}$. Then $\sigma_{-x_{0}} f$ is in $K_{0}$

Received by the editors October 26, 1959.

1 For details on this topology, see $\mathrm{R}$. Thom, Les singularités des applications différentiables, Ann. Inst. Fourier, Grenoble vol. 6 (1956) pp. 43-87. 
and $f=\sigma_{x} g, g \in K_{0}$. Furthermore, the decomposition is unique. Indeed if $\sigma_{x} g=\sigma_{y} h$ then $g h^{-1}=\sigma_{y-x}$ is a translation. Since $g h^{-1} \in K_{0}, y=x$, $g=h$. That the topology on $K$ is the topology of the product space is easily established.

Now let

$$
H=\left\{g \in K_{0} \mid \frac{\partial g_{i}}{\partial x_{k}}=\delta_{k}^{i}\right\} .
$$

$H$ is then a closed, invariant subgroup and

LEMma 2. $K_{0}$ decomposes topologically into $\mathrm{Gl}(n, R) \times H$ where $\mathrm{Gl}(n, R)$ is the group of non-singular matrices on $E^{n}$.

Proof. Consider the homomorphism $\phi: K_{0} \rightarrow \operatorname{Gl}(n, R)$ defined by $\phi(f)=\left(\left(\partial f_{i} / \partial x_{k}\right)(0)\right) . \phi$ is continuous on $K_{0}$ since we have demanded close partial derivatives. If $\alpha \in \mathrm{Gl}(n, R)$ then $\phi(\alpha)=\alpha$. If $f \in K_{0}$ consider the element $\phi(f)^{-1} f$ under $\phi$. We have

$$
\phi\left(\phi(f)^{-1} f\right)=\phi\left(\phi(f)^{-1}\right) \phi(f)=\phi(f)^{-1} \phi(f)=I
$$

the identity matrix, and $\phi(f)^{-1} f$ is in the kernel of $\phi$ which is clearly $H$, and $f=\phi(f) g, g \in H$. If $\alpha g=\beta h, \alpha, \beta \in \operatorname{Gl}(n, R), g, h \in H$ then $\beta^{-1} \alpha=h g^{-1}$ and since $h g^{-1} \in H, \beta^{-1} \alpha=I$ and consequently $\alpha=\beta$ and $g=h$. Again the product topology of $K_{0}$ can be shown to be the product topology on $\mathrm{Gl}(n, R)$ and $H$.

Lemma 3. The group $H$ is contractible to a point.

Proof. For $0<t<\infty$, denote by $L_{t}$ the transformation $L_{t} x=t x$. Then we define the homotopy $\Phi: H \times[0,1] \rightarrow H$ by

$$
\Phi(f, t)=\left\{\begin{array}{cl}
L_{1 / t} f L_{t}, & 0<t \leqq 1, \\
I, & t=0 .
\end{array}\right.
$$

We have $\Phi(f, 1)=f$ and $\Phi(f, 0)=I$, thus if $\Phi$ is continuous, $\Phi$ contracts $H$ to $I$. First we see that $\Phi$ is continuous on $H \times[0,1]$ since on this set $\Phi$ amounts to a continuous system of inner automorphisms and these are continuous since the group operations are continuous.

Now let $f \in H$, we will show that $\Phi$ is continuous at $(f, 0)$. Suppose we are given a neighborhood $V_{r, \epsilon}$ of $I$ as given by (1), (we suppose $r>1$ ). We must show that there exists a neighborhood, $U$, of $f$ in $H$

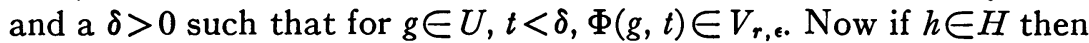
by the theorem of the mean we have

$$
h_{i}(x)=x_{i}+\sum_{k=1}^{n} c_{i}^{k}(h ; x) x_{k}
$$


and $c_{i}^{k}(h ; x)$ tends to 0 with $x$, (in fact $c_{i}^{k}(h ; x)=\left(\partial h_{i} / \partial x_{k}\right)\left(\theta_{k}^{i} x\right)$ $\left.-\delta_{i}^{k}, 0<\theta_{k}^{i}<1\right)$. Furthermore, denoting by $h^{t}$ the transformation $\Phi(h, t), t>0$ we have $\left(\partial h_{i}^{i} / \partial x_{j}\right)(x)=\left(\partial h_{i} / \partial x_{j}\right)(t x)$. Then we first select a neighborhood $U_{1}$ of $f$ so that for

$$
g \in U_{1}, \quad\|x\| \leqq r, \quad\left|\frac{\partial g_{i}}{\partial x_{k}}(x)-\frac{\partial f_{i}}{\partial x_{k}}(x)\right|<\frac{\epsilon}{2 r n} .
$$

Since $\left(\partial f_{i} / \partial x_{k}\right)(0)=\delta_{k}^{i}$ we can choose $\eta>0$ so that $\|x\|<\eta$ implies $\left|\left(\partial f_{i} / \partial x_{k}\right)(x)-\delta_{\boldsymbol{k}}^{i}\right|<\epsilon / 2 r n$. Choose $\delta_{1}>0$ so that for $0<t<\delta_{1},\|x\| \leqq r$ we have $\|t x\|<\eta$. Under these conditions we obtain for $g \in U_{1}$, $0<t<\delta_{1},\|x\| \leqq r$

$$
\begin{aligned}
\left|\frac{\partial g_{i}^{t}}{\partial x_{k}}(x)-\delta_{k}^{i}\right| & =\left|\frac{\partial g_{i}}{\partial x_{k}}(t x)-\delta_{k}^{i}\right| \\
& \leqq\left|\frac{\partial g_{i}}{\partial x_{k}}(t x)-\frac{\partial f_{i}}{\partial x_{k}}(t x)\right|+\left|\frac{\partial f_{i}}{\partial x_{k}}(t x)-\delta_{k}^{i}\right| \\
& <\frac{\epsilon}{r n} .
\end{aligned}
$$

Since the inequality holds for all $x$ for which $\|x\| \leqq r$ it also holds for $\theta x, 0<\theta<1,\|x\| \leqq r$ and we conclude that for $g \in U_{1},\left|c_{i}^{k}(g ; t x)\right|$ $<\epsilon / r n$. Then

$$
\begin{aligned}
\left|g_{i}^{t}(x)-x_{i}\right| & =\left|\sum_{j=1}^{n} c_{i}^{j}(g ; t x) x_{j}\right| \\
& \leqq \sum_{j=1}^{n}\left|c_{i}^{j}(g ; t x)\right|\left|x_{j}\right| \\
& <\epsilon .
\end{aligned}
$$

Hence $\Phi$ maps $U_{1} \times\left[0, \delta_{1}\right)$ into $V_{r, \epsilon}$ and $\Phi$ is continuous at $(f, 0)$ and the lemma is proved.

Proof of Theorem I. It follows from Lemmas $1,2,3$ that $K=E^{n} \times \mathrm{Gl}(n, R) \times H$ as a topological space. Now $\mathrm{Gl}(n, R)$ can be topologically decomposed as the product of the orthogonal group and a euclidean space. Then $K$ is topologically the product $O_{n} \times A$ where $A$ is a contractible space, and Theorem I follows readily.

Corollary I. Let $B=\left\{B, p, X, E^{n}, K\right\}$ be a fibre bundle in the sense of Steenrod, [2, p. 8], with fibre $E^{n}$, group $K$ and base space $X$ a locally finite polyhedra. Then $B$ is equivalent in $K$ to a bundle with group $O_{n}$. 
Proof. According to $[2$, p. 36] it suffices to show that the bundle $\Theta^{\prime}$ associated to $B$ with fibre $K / O_{n}$ has a cross-section. It follows from Theorem I that $\Pi_{i}\left(K / O_{n}\right)=0, i \geqq 0$. Since $X$ is a locally finite polyhedra it follows in the usual way that we can define a crosssection inductively on the $k$-dimensional skeleton of $X$ and obtain the corollary.

II. We consider now the sphere, $S^{n}$, and the group $K$ of homeomorphisms $f$ of $S^{n}$ such that $f$ and $f^{-1}$ are of class $C^{1}$ in the usual differentiable structure of $S^{n}$. We suppose $S^{n}$ covered by two coordinate neighborhoods, $U_{1}$ and $U_{2}, U_{1}=S^{n} \backslash\left\{x_{0}\right\}, U_{2}=S^{n} \backslash\left\{-x_{0}\right\}$. If $(x)$ are the coordinates on $U_{1}$ and $(y)$ the coordinates on $U_{2}$ we take as coordinate transformations

$$
y_{i}=\frac{x_{i}}{\sum_{k=1}^{n} x_{k}^{2}}
$$

$K$ is topologized with the usual $C^{1}$-topology (cf. the reference in footnote 1).

THEOREM II. If $O_{n+1}$ is the orthgonal group then $i_{*}: \Pi_{k}\left(O_{n+1}\right) \rightarrow \Pi_{k}(K)$ is an isomorphism into where $i_{*}$ is induced by the injection $i: O_{n+1} \rightarrow K$.

The proof follows

Lemma 4. Let $K_{0}$ denote the subgroup of $K$ which holds $x_{0}$ fixed. Then $i: O_{n} \rightarrow K_{0}$ induces an isomorphism $i_{*}$ of $\Pi_{k}\left(O_{n}\right)$ into $\Pi_{k}\left(K_{0}\right)$ for each $k{ }^{2}$

Proof. We suppose $x_{0}$ to have coordinates $(0, \cdots, 0)$ in $U_{1}$. Define the continuous homomorphism $\phi: K_{0} \rightarrow \mathrm{Gl}(n, R)$ by

$$
\phi(g)=\left(\frac{\partial g_{i}}{\partial x_{k}}(0)\right) .
$$

For each $g, \phi(g)$ can be expressed uniquely and continuously as the product of an orthogonal matrix $\psi(g)$ and a triangular matrix $\pi(g)$, $\phi(g)=\psi(g) \pi(g)$. Then $\psi: K_{0} \rightarrow O_{n}$ and for $h \in O_{n}, \psi(h)=h$ so that $\psi$ is a retraction of $K_{0}$ onto $O_{n}$. The lemma then follows immediately.

Proof of Theorem II. It is easily seen that the map $f: K \rightarrow S_{n}$, $f(g)=g\left(x_{0}\right)$ is open (since $f$ is open on $O_{n+1}$ ) and consequently that $K / K_{0}$ is canonically homeomorphic to $S^{n}$. Furthermore, the local cross-section of $O_{n+1}$ over $S^{n}$ provides a local cross-section for $K$ over

$2 O_{n}$ can be imbedded in $K_{0}$ so that its action is that of $O_{n}$ on the coordinate neighborhood $U_{1}$. 
$S^{n}$, and $K$ is then a fibre bundle over $S^{n}$. Consequently we have the following commutative diagram of exact sequences

$$
\begin{aligned}
& \cdots \rightarrow \Pi_{k}\left(O_{n}\right) \rightarrow \Pi_{k}\left(O_{n+1}\right) \rightarrow \Pi_{k}\left(S^{n}\right) \rightarrow \Pi_{k-1}\left(O_{n}\right) \rightarrow \Pi_{k-1}\left(O_{n+1}\right) \rightarrow \cdots \\
& \begin{array}{lllll}
\downarrow i_{*} & \downarrow i_{*} & \downarrow j_{*} & \downarrow i_{*} & \downarrow i_{*}
\end{array} \\
& \cdots \rightarrow \Pi_{k}\left(K_{0}\right) \rightarrow \Pi_{k}(K) \rightarrow \Pi_{k}\left(S^{n}\right) \rightarrow \Pi_{k-1}\left(K_{0}\right) \rightarrow \Pi_{k-1}(K) \rightarrow \cdots
\end{aligned}
$$

where $j_{*}$ is the identity. It is easily seen that $\operatorname{Ker}\left(i_{*}: \Pi_{k}\left(O_{n+1}\right) \rightarrow \Pi_{k}(K)\right)$ is the image under $\Pi_{k}\left(O_{n}\right) \rightarrow \Pi_{k}\left(O_{n+1}\right)$ of $\operatorname{Ker}\left(i_{*}: \Pi_{k}\left(O_{n}\right) \rightarrow \Pi_{k}\left(K_{0}\right)\right)$. Since this last kernel is $O$ the theorem follows.

\section{REFERENCES}

1. D. Montgomery and L. Zippin, Topological transformation groups, New York, Interscience Publishers, 1955.

2. N. Steenrod, The topology of fibre bundles, Princeton, Princeton University Press, 1951.

UNIVERSITY OF NOTRE DAME

\section{A NOTE ON GAUSS' FIRST PROOF OF THE QUADRATIC RECIPROCITY THEOREM}

\section{CARLITZ}

We assume that the reader is familiar with Mathews' exposition $[1$, pp. 45-50] of the inductive proof of the reciprocity theorem. There are three main cases:

I. $p R q$,

II. $p N q, q \equiv 3(\bmod 4)$,

III. $p N q, q \equiv 1(\bmod 4)$.

In I we have $e^{2}-p=q^{f}$, in II we have $e^{2}+p=q^{f}$. In III we have first the lemma which asserts the existence of a prime $p^{\prime}<q$ such that $q N p^{\prime}$. This implies $p^{\prime} N q$, so that $p p^{\prime} R q$ and so $e^{2}-p p^{\prime}=q f$. In each of the cases I and II it is necessary to treat two sub-cases; in case III there are four sub-cases. Thus in all there are eight cases to consider.

We should like to point out in this note that it is possible to handle all cases simultaneously by introducing a little notation. To begin with, we define

Received by the editors September 22, 1959. 